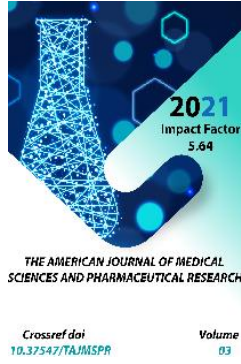

\title{
Effective Treatment Of Afthous Stomatitis In The Practice Of
} A Doctor

\section{Sharifa Abdupattoevna Borotova}

Assistant, Department Of Training And Retraining Of A Family Doctor, Andijan State Medical Institute, Uzbekistan

\section{ABSTRACT}

Aphthous stomatitis is a focal inflammation of the oral mucosa, in which round ulcers (aphthae or erosion) form. Aphthae affect the inner surface of the cheeks, palate, tongue, become covered with a gray or yellowish coating, cause pain and discomfort. Usually the disease proceeds chronically, from time to time exacerbates, after 7-10 days it goes into remission.

\section{KEYWORDS}

Aphthous stomatitis, oral membranes, round ulcers (aphthae or erosion). the inner surface of the cheeks, palate, tongue, painful sensations, discomfort, stage of remission.

\section{INTRODUCTION}

According to ICD-10, aphthous stomatitis is assigned the K.12 code. Inside the code, there are three clarifying diagnoses, including K.12.0
- "recurrent oral aphthae", which also includes aphthous ulcers. 


\section{MATERIALS AND METHODS}

According to the form of the course, acute and chronic aphthous stomatitis is distinguished. The first is characterized by the appearance of ulcers and severe hyperemia - edema, redness of mucous membranes. Severe pain occurs, especially when eating or talking. Submandibular lymph nodes may increase, body temperature may rise.

\section{RESULT AND DISCUSSION}

Chronic recurrent aphthous stomatitis can be the result of improper or untimely treatment, as well as the inability of the immune forces to cope with the disease. It is distinguished by periodic exacerbations. Without exacerbation, symptoms can be erased, completely absent.

Average. Up to $5 \mathrm{aft}$, the course of treatment takes up to 3 weeks. Soreness is quite pronounced, there is an increase in lymph nodes, relapses up to 2 times a year;

Heavy. Multiple aphthae appear, severe soreness. An increase in temperature occurs, symptoms of general intoxication appear. Treatment takes up to a month, relapses occur up to 6 times a year.

Also, according to the form of the course, the following types of inflammatory diseases are distinguished:

Fibrous. Microcirculation of blood in the upper layer of the mucous membrane is disturbed, aphthae appear covered with a fibrous film (plaque). These ulcers heal completely in up to 14 days. The disease affects the mucous membrane of the lips, the lateral surfaces of the tongue, transitional folds. Such stomatitis recurs up to 3 times a year.
Necrotic. The epithelium is destroyed, the mucosal area dies off. Replacement of tissues with normal epithelium takes 14 to 30 days. Such aphthous stomatitis is not accompanied by acute pain, it is usually observed in patients with severe diseases, including blood pathologies.

Grandular. There is a defeat of the ducts of the small salivary glands. In this regard, aphthae are formed close to the glands, there is a desiccation of the mucous membranes of the oral cavity due to a decrease in saliva production. Ulceration is painful, healing occurs within $1-3$ weeks.

Scarring. Damage to the cavity of the salivary glands leads to the involvement of connective tissue in inflammation. Aphthae are observed both at the location of the glands, and on the mucous membranes of the pharynx and palate. The disease turns into large painful ulcers (up to $1.5 \mathrm{~cm}$ ). Healing takes up to 12 weeks, after the acute process, pronounced scars remain;

Deforming. The most severe form of the disease, which provokes changes in connective tissue. Aphthae heal extremely slowly, which is accompanied by deformation of the palate, lips, sometimes there is a narrowing of the mouth gap (if aphthae were observed in the corners of the mouth).

Usually, 1-2 days before the appearance of aphtha, areas of the mucous membrane with increased sensitivity are found, and a burning sensation may occur. The aphthae themselves are round, have clear boundaries, covered with a gray or yellowish coating. Their size, as a rule, does not exceed $1 \mathrm{~cm}$, the mucous membranes around them turn red. Such areas of erosion heal in up to 2 weeks without scarring. But in 1 case out of 10 , the diameter of the ulcers is 
more than $1 \mathrm{~cm}$, they affect deeper areas of tissues, the boundaries of the pathological area may look raised. Healing in this case lasts up to 6 weeks, after which a scar is formed. Aphthous stomatitis is characterized by damage to the mucous membranes of the cheeks, the inner side of the lips, soft palate, tonsils, and lateral surfaces of the tongue. This is due to the absence of keratinization of the epithelium in these areas. Much less often, aphthae appear on the hard palate, the back of the tongue, and the gums. The main goal of the treatment of aphthous stomatitis is to completely get rid of the disease, or at least reduce the frequency of relapses to a minimum. Therapy is aimed at relieving inflammation, relieving unpleasant symptoms, accelerating the recovery processes of the mucous membrane.

For each specific case, the doctor will develop a set of measures. The main ones include local and systemic drug treatment. Antiseptic rinses can be carried out using medications or mouth rinses, which include antibacterial components. An alternative to rinsing is the use of a spray. Typically, the treatment regimen includes 2-3 rinsing sessions for 1 minute immediately after brushing your teeth.

Topical treatment methods include the application of gels with anti-inflammatory and analgesic effects.

Occlusive agents can reduce pain and speed up the healing of mucous membranes. They form an insoluble film on the ulcer and protect the affected area from adverse factors.

Local glucocorticoid therapy is used against the background of immune diseases, as well as when standard measures are ineffective. They allow you to eliminate pain and quickly relieve inflammation, shorten the healing period. Such funds are used only according to indications, dispensed with a prescription. In some cases, it is advisable to inject the drug under the base of the ulcer, this is done by the doctor.

Preparations for epithelialization are used after the removal of acute inflammation. The specialist will prescribe a gel with an analgesic and healing effect, usually 5-6 days after the start of complex therapy.

Local laser therapy can relieve pain, speed up the healing process, and minimize the risk of relapse. Systemic treatment of aphthous stomatitis in adults involves taking the following drugs: antihistamines (anti-allergic, anti-edema);

Glucocorticoids (anti-inflammatory, analgesic effect);

Immunomodulators (to stimulate the defenses, accelerate recovery).

\section{CONCLUSION}

And if antiallergic drugs can be recommended to any patient even in the absence of information about the exact causes of stomatitis, then the rest of the drugs are prescribed only according to indications: with an acute severe course of the disease, frequent relapses, the presence of severe systemic pathologies. An additional method of treatment is vitamin therapy - taking vitamins C, group B.

In addition to the main course of treatment, all patients, without exception, are advised to adhere to a hypoallergenic diet, refuse to take too hot drinks and dishes, spicy, irritating mucous food. It is better to give preference to a toothpaste without sodium lauryl sulfate, this component can provoke the disease. 
It is important to continue oral hygiene, even if L.: "Selkhozizdat" 1985

it is difficult. To make brushing your teeth easier, choose a soft toothbrush. If the disease recurs often, it is necessary to pay attention to the general state of health, timely treat teeth and gums, replace fillings and dental structures.

\section{Features of treatment in children}

The dentist will tell you how to treat aphthous stomatitis in a child. You can refer to him in the direction of the pediatrician or on your own, finding characteristic ulcers in the mouth. The treatment regimen coincides with that used in the therapy of adult patients, but there are some differences: children under a certain age cannot rinse the mouth, therefore, preference is given to drugs for application to mucosal ulcers. Otherwise, the therapy regimen is developed individually, taking into account the severity, symptoms of the disease, the frequency of relapses, the presence or absence of concomitant ailments in the child. Symptomatic therapy can be used to quickly relieve the baby's condition.

\section{REFERENCES}

1. Pediatrics. V.A. Belousov, A.D. Pevzner Uzbek citizen Tashkent city "Mediuina" Uzbek SSR $1972 \mathrm{y}$.

2. A textbook on nutrition for a healthy child. Prof. A.V. Mazurin. Leningrad "Mediuina" 1982

3. Propedeutics of childhood diseases. A.F. Tour. Leningrad "Mediuina" 1967

4. Kodirov E. "Histology" T .: "Teacher" 1994

5. Alimov D.A. Histology va Embryology. T. "Teacher" 1996

6. Sidorova M.V. Davidova Workshop on anatomy with the basics of histology. 